\title{
Declaração de óbito: preenchimento pelo corpo clínico de um hospital universitário
}

Luan Lucena ${ }^{1}$, Gustavo Henrique Bocalon Cagliari ${ }^{2}$, Julio Tanaka ${ }^{3}$, Elcio Luiz Bonamigo ${ }^{4}$

\section{Resumo}

Esta pesquisa analisou o preenchimento das declarações de óbito em um hospital universitário. $O$ estudo objetivou avaliar, em prontuários médicos, as declarações de óbito dos anos 2009 e 2011 e as dificuldades dos médicos do corpo clínico em preenchê-las. Realizou-se pesquisa documental e aplicação de questionários aos médicos. Das 528 declarações de óbito analisadas, 265 (50,18\%) estavam incompletamente preenchidas. Dos 34 médicos participantes, 34,14\% referiram como principal dificuldade de preenchimento as mortes sem assistência médica; $26,47 \%$ consideraram ter tido um aprendizado ruim durante a graduação e somente $50 \%$ conheciam o documento que orienta o preenchimento da declaração. Como conclusão, infere-se a necessidade de aprimorar o ensino na graduação acerca da declaração de óbito, bem como incentivar a atualização médica sobre este importante aspecto.

Palavras-chave: Declaração de óbito. Epidemiologia. Causas de morte. Indicadores de morbimortalidade.

\section{Resumen}

\section{Certificado de defunción: cumplimentación por el cuerpo médico en un hospital universitario}

Esta investigación analizó la cumplimentación de los certificados de defunción en un hospital universitario. El objetivo fue evaluar, en registros médicos, los certificados de defunción de los años 2009 y 2011 y las dificultades para el personal médico en cumplimentarlos. Se realizó la investigación documental y encuestas a los médicos. De los 528 certificados de defunción analizados, 265 (50,18\%) fueron incompletamente cumplimentados. De los 34 médicos participantes, el 34,14\% apuntó que la principal dificultad en la cumplimentación fueron las muertes sin asistencia médica; el $26,47 \%$ consideró escasa su aprendizaje durante la graduación y; sólo el $50 \%$ conocía el documento guía que orienta la cumplimentación del certificado. En conclusión, se infiere la necesidad de perfeccionar la enseñanza en el pregrado acerca del certificado de defunción, así como promover la actualización médica sobre este importante aspecto.

Palabras-clave: Certificado de defunción. Epidemiología. Causas de muerte. Indicadores de morbimortalidad.

\section{Abstract \\ Death certificate: filling by the medical staff of a university hospital}

This investigation analyzed the contents from death certificates filled in by a university hospital. The study aimed to evaluate in medical records the death certificates of the years 2009 and 2011, in the database, and the difficulties of the medical clinical staff to fill them in. A documental research was done as well as interviews with the doctors. In the 528 death certificates analyzed, it was found that 265 (50.18\%) were incomplete. From the 34 clinical physicians who answered the questionnaire, $34.14 \%$ reported that deaths without medical assistance constitute the major difficulty in filling the document; $26.47 \%$ mentioned that they believe their learning during the undergraduate course was not good, and only half of them knew the guideline to fill in the certificate. In conclusion, it is inferred the need to improve the teaching during de undergraduate course and encourage the updating of doctors when it comes to this important aspect.

Key words: Death certificates. Epidemiology. Cause of death. Indicators of morbidity and mortality.

Aprovação CEP Unoesc nº 40194

1. Graduando luanlucena@icloud.com 2. Graduando gustavocagliari@yahoo.com.br 3. Mestre julioakeotanaka@yahoo.com.br 4. Doutor elcio.bonamigo@unoesc.edu.br - Universidade do Oeste de Santa Catarina (Unoesc) Campus de Joaçaba, Joaçaba/SC, Brasil.

\section{Correspondência}

Elcio Luiz Bonamigo - Rua Francisco Lindner, 310 CEP 89600-000. Joaçaba/SC, Brasil.

Declaram não haver conflito de interesse. 
As informações provenientes das declarações de óbito (DO) e do Sistema de Informações sobre Mortalidade (SIM) ${ }^{1}$ são fundamentais para o planejamento de ações públicas, o desenvolvimento da gestão da saúde e a realização de estudos epidemiológicos. A implantação do SIM pelo Ministério da Saúde (MS) ${ }^{2}$, em 1975, que utiliza os dados constantes nas DO, representou considerável avanço na produção de informações sobre mortalidade no Brasil. Ressalte-se que a criação desse sistema foi motivada pela necessidade de informações completas acerca dos óbitos ocorridos no país. Essas informações permitem realizar análises e estudos especiais para detectar e corrigir tanto o sub-registro de dados como as deficiências de qualidade da informação ${ }^{3}$.

A utilização da DO padronizada, para óbitos fetais e não fetais, implantada nacionalmente pela Lei 6.015, de 31 de dezembro de $1973^{4}$, permitiu a uniformização nacional dos dados e facilitou a disponibilização de informações de interesse para o setor saúde. Considerado o instrumento padronizado que alimenta o SIM, a DO possibilita a obtenção de estatísticas de melhor qualidade sobre as causas de morte no Brasil. Contudo, isto supõe o preenchimento correto, para que a proporção dos óbitos por causas mal definidas, decorrentes de preenchimento incompleto ou inadequado, seja reduzida. Assim, declarações de óbito adequadamente preenchidas são essenciais para aprimorar a qualidade dos dados estatísticos sobre a mortalidade da população de um país.

O preenchimento das declarações de óbito constitui responsabilidade ética e jurídica ${ }^{4}$ do médico que assina o documento, como previsto nos artigos 82 e 83 do Código de Ética Médica de $2009^{5}$. Porém, é possível que o preenchimento possa estar sendo interpretado como exigência meramente legal e burocrática, sem receber a devida atenção dos responsáveis quanto à importância dos dados a serem registrados. Tendo em vista tal situação, o principal objetivo desta pesquisa foi analisar o preenchimento das declarações de óbito em um hospital universitário e obter informações dos médicos sobre o conhecimento e as dificuldades de preenchimento, buscando contribuir para a superação desses entraves e, consequentemente, para a melhoria dos indicadores de mortalidade.

\section{Método}

Trata-se de estudo quantitativo, retrospectivo e transversal nas declarações de óbito de pacientes falecidos no Hospital Universitário Santa Terezinha, em Joaçaba/SC, durante os anos de 2009 e 2011. A escolha desses anos para a realização do estudo deveu-se à existência de outro estudo desenvolvido no mesmo hospital ${ }^{6}$, concluído em 2010: um trabaIho de conclusão de curso que encontrou elevada frequência de inadequações no preenchimento das declarações de óbito, sobretudo quanto ao uso de termos inadequados, cujos resultados foram levados ao conhecimento dos principais responsáveis da instituição. Desta forma, presumiu-se que as declarações de óbito do ano 2011, posteriores ao citado estudo, estariam mais adequadamente preenchidas do que as precedentes, de 2009. A coleta foi realizada na Secretaria de Saúde do Município de Joaçaba/ SC, onde as DO são armazenadas, sendo excluídas da pesquisa as originárias de outros hospitais e/ou localidades.

Os dados foram colhidos nas declarações de óbito originais, diretamente encaminhadas pelo hospital à secretaria de saúde, previamente a quaisquer alterações posteriormente feitas pelos responsáveis para fins de arquivamento e organização dos dados. Foram consideradas declarações de óbito corretas as que apresentavam todos os itens (de II a VII) adequadamente preenchidos. A coleta foi aprovada e supervisionada pelo responsável do setor na secretaria municipal de saúde e pelo comitê de ética em pesquisa.

Com o objetivo de identificar e analisar os problemas relatados pelos médicos no preenchimento das declarações de óbito foi aplicado, aos participantes do corpo clínico do hospital, um questionário com 15 questões fechadas. Para a análise dos dados, foram utilizadas planilhas do programa Excel e o sistema computacional BioEstat $4.0 \mathrm{com}$ nível de significância de $p<0,05$.

\section{Resultados}

Em relação ao preenchimento, das 298 declarações de óbito de 2009 analisadas, 205 (68,79\%) apresentavam preenchimento completo e 93 (31,21\%), incompleto. Em 2011, das 230 DO analisadas $58(25,21 \%)$ apresentavam preenchimento completo e 172 (74,79\%), incompleto $(p<0,001)$. Em números totais, foram analisadas 528 declarações de óbito, $263(49,81 \%)$ completamente preenchidas e $265(50,18 \%)$ com preenchimento incompleto (Tabela 1). 
Tabela 1. Preenchimento das declarações de óbito nos anos de 2009 e 2011

\begin{tabular}{|c|c|c|c|}
\hline \multirow{2}{*}{ Preenchimento } & 2009 & 2011 & Anos (\%) \\
\hline Completo & $\mathrm{n}(\%)$ & $\mathrm{n}(\%)$ & $\mathrm{n}(\%)$ \\
\hline Incompleto & $205(68,79)$ & $58(25,21)$ & $263(49,81)$ \\
\hline Total (\%) & $93(31,21)$ & $172(74,79)$ & $\mathbf{5 2 8 ( 1 0 0 )}$ \\
\hline
\end{tabular}

$(p<0,001)$

Ao correlacionar os médicos subscritores das declarações de óbito com os anos de 2009 e 2011, em um modelo que os dividiu nas categorias médico assistente, substituto, médico legista (IML), outros e não preenchidas, verificou-se que 267 (89,60\%) DO foram assinadas em 2009 por médicos assistentes, $13(4,30 \%)$ por médicos substitutos, 3 (1\%) por médicos legistas, 12 (4,10\%) por outros médicos e $3(1 \%)$ não estavam preenchidas. Em 2011, 155 (67,40\%) fo- ram assinadas por médicos assistentes, 24 (10,42\%) por médicos substitutos, 12 (5,22\%) por médicos legistas, 12 (5,22\%) por outros médicos e $27(11,74 \%)$ não estavam preenchidas. As variações mostraram significância estatística ( $p<0,001)$. Em números totais, 422 (79,92\%) DO foram assinadas por médicos assistentes, 37 (7\%) por médicos substitutos, 15 $(2,84 \%)$ por legistas, 24 (4,54\%) por outros médicos e $30(5,68 \%)$ não estavam preenchidas (Tabela 2$)$.

Tabela 2. Categorias dos médicos assinantes das declarações de óbito nos anos de 2009 e 2011

\begin{tabular}{|c|c|c|c|}
\hline \multirow{2}{*}{ Médico } & \multicolumn{3}{|c|}{ Anos (\%) } \\
\hline Assistente & 2009 & 2011 & Total \\
\hline Substituto & $\mathrm{n}(\%)$ & $\mathrm{n}(\%)$ & $422(79,92)$ \\
\hline Legista (IML) & $267(89,60)$ & $155(67,40)$ & $37(7)$ \\
\hline Outros & $13(4,30)$ & $24(10,42)$ & $15(2,84)$ \\
\hline Não preenchidas & $3(1,00)$ & $12(5,22)$ & $24(4,54)$ \\
\hline Total (\%) & $3(4,10)$ & $27(11,74)$ & $30(5,68)$ \\
\hline
\end{tabular}

$(p<0,001)$

O uso de termos vagos ou inadequados foi visto em 19 (6,37\%) das 298 DO de 2009, sendo a "parada cardiorrespiratória" (10 - 3,35\%) e "falência múltipla de órgãos" (3 - 1\%) os mais utilizados. No ano 2011, das 230 DO 15 (6,52\%) apresentavam termos vagos ou inadequados, sendo "parada cardiorrespiratória" (5 - 2,17\%) o mais frequente. No total, das 528 DO examinadas, 34 (6,43\%) apresentavam termos vagos ou inadequados, prevalecendo "parada cardiorrespiratória" em 15 (2,84\%).

Dos 54 médicos do corpo clínico, 34 (62,9\%) participaram da pesquisa. Em relação ao conhecimento sobre o documento do Conselho Federal de Medicina (CFM)/Ministério da Saúde intitulado A declaração de óbito: documento necessário e importante, verificou-se que dos 34 (100\%) médicos participantes 16 (47,0\%) o conheciam, 14 (41,2\%) o desconheciam e $4(11,8 \%)$ abstiveram-se de responder.
Quanto ao uso de termos não recomendados - como "parada cardíaca", "parada cardiorrespiratória" ou "falência múltipla de órgãos" - para o preenchimento de causa básica nas declarações de óbito, verificou-se que 13 (38,2\%) dos 34 médicos participantes os utilizavam, 17 (50\%) não os utilizavam e 4 $(11,8 \%)$ não responderam.

Ao se indagar os médicos sobre as maiores dificuldades no preenchimento das declarações de óbito, em uma questão que permitia assinalar mais de uma causa, verificou-se que 14 (34,14\%) tinham dificuldade no preenchimento de óbitos sem assistência médica; 10 (24,39\%), no preenchimento da causa básica, intermediária e imediata ou terminal da morte; $5(12,19 \%)$, no preenchimento de óbitos de causa violenta; 3 (7,31\%), no preenchimento de declarações de recém-nascidos (com menos de 500 gramas); 3 (7,31\%), nas amputações de membros; 2 (9,75\%), em outras causas e $4(9,75 \%)$ não responderam (Tabela 3 ). 
Tabela 3. Principais dificuldades no preenchimento da declaração de óbito

\begin{tabular}{|lc|}
\hline \multicolumn{1}{|c|}{ Dificuldade $p /$ preenchimento DO } & $\mathbf{N}^{\circ}(\%)$ \\
\hline Óbito em recém-nascidos & $3(7,31)$ \\
\hline Óbito de causa violenta & $5(12,19)$ \\
Óbito sem assistência & $14(34,14)$ \\
Relatório sobre amputação de membros & $3(7,31)$ \\
$\begin{array}{l}\text { Causa básica, intermediária e imediata ou } \\
\text { terminal }\end{array}$ & $10(24,39)$ \\
Outra causa & $2(4,87)$ \\
Não responderam & $4(9,75)$ \\
\hline
\end{tabular}

Quanto ao conhecimento sobre o preenchimento da declaração de óbito em relação ao tempo de formação médica, os resultados evidenciaram que 13 (38,23\%) médicos com tempo de formação até 20 anos consideraram seu aprendizado bom ou muito bom; 11 (32,25\%), aceitável e 3 (8,82\%), ruim. Com o tempo de formação entre 21 e 45 anos, 3 $(8,82 \%)$ dos médicos consideraram seu aprendizado bom; 3 (8,82\%), aceitável e apenas 1 (2,94\%) muito ruim $(p=250)$. Em números totais, $16(47,02 \%)$ médicos consideraram seu aprendizado muito bom ou bom; $14(41,18 \%)$, aceitável e $4(11,76 \%)$, ruim ou muito ruim (Tabela 4 ).

Tabela 4. Conhecimento atual e tempo de formação médica

\begin{tabular}{|c|c|c|c|}
\hline \multirow{2}{*}{ Conhecimento atual } & \multicolumn{2}{|c|}{ Tempo de formação } & Total \\
& Até 20 anos & $21-45$ anos & $\mathrm{n}(\%)$ \\
\hline Muito bom & $\mathrm{n}(\%)$ & $\mathrm{n}(\%)$ & $1(2,94)$ \\
\hline Bom & $1(2,94)$ & 0 & $15(44,12)$ \\
\hline Aceitável & $12(35,29)$ & $3(8,82)$ & $14(41,18)$ \\
Ruim & $11(32,35)$ & $3(8,82)$ & $3(8,82)$ \\
\hline Muito ruim & $3(8,82)$ & 0 & $1(2,94)$ \\
\hline Total (\%) & 0 & $1(2,94)$ & $\mathbf{3 4}(\mathbf{1 0 0})$ \\
\hline
\end{tabular}

$(p=0,250)$

\section{Discussão}

Menos da metade das declarações de óbito examinadas neste estudo $(49,81)$ foi preenchida de forma completa (Tabela 1). Estudo realizado no Rio de Janeiro com 806 declarações de óbito ${ }^{7}$ apresentou resultado semelhante: somente $52,3 \%$ das DO examinadas estavam corretamente preenchidas. No entanto, outro estudo desenvolvido em Belo Horizonte ${ }^{8}$ mostrou número bem mais elevado de preenchimentos incompletos. Foram examinadas 282 DO de duas instituições: 141 (50\%) provinham do serviço de cardiologia do Hospital de Base (HB) e 141 (50\%), do Serviço de Verificação de Óbitos (SVO). Erros ou falhas de preenchimento ocorreram em $100 \%$ das DO provenientes do grupo HB e em $88,08 \%$ do grupo SVO. Resultados similares também foram encontrados em estudo realizado em Belém, no qual $98,7 \%$ das DO apresentavam pelo menos um equívoco de preenchimento ${ }^{9}$. Níveis elevados de causas mal definidas colocam em discussão a qualidade das informações das declarações de óbi- tos e a consistência do uso desses dados para certas unidades geográficas ${ }^{10}$.

Assim como ocorreu no presente estudo, os problemas no preenchimento costumam aparecer com clareza em todos os itens existentes da declaração de óbito, desde os dados de identificação, história gestacional da mãe, até mesmo nas causas de morte, sendo reforçados quando óbitos ocorrem sem assistência médica ?

$\mathrm{Na}$ instituição em que se realizou o presente trabalho ficou evidente que a divulgação interna, em $2010{ }^{6}$, dos resultados de um trabalho de conclusão do curso de medicina, que apontou falhas de preenchimento das DO e teve em sua banca a participação de um dos responsáveis pela UTI, não surtiu o efeito desejado na melhora no preenchimento. Ao se examinar os resultados de 2009 verificou-se que das 298 declarações de óbito existentes 205 (68,9\%) foram corretamente preenchidas. No ano 2011, ao contrário do que se esperava, das 230 declarações de óbito apenas $58(25,21 \%)$ foram corretamente preenchidas (Tabela 1 ). 
Para tentar identificar as causas da piora do preenchimento foram examinadas as categorias profissionais dos médicos responsáveis pelo preenchimento nos dois períodos. Observou-se em 2009 que $89,60 \%$ das DO foram preenchidas por médicos assistentes, índice que em 2011 decaiu para 67,40\% $(p<0,001)$. Assim, inferiu-se que o preenchimento da DO por menor número de médicos assistentes possa ter contribuído para a piora do preenchimento das DO no ano 2011 (Tabela 2).

Diversos fatores podem contribuir para o preenchimento incorreto da DO, tais como a assistência médica prestada no momento do óbito, o tipo de serviço utilizado pelo paciente no momento do óbito (emergência, UTI, obstetrícia, clínica médica) e, ainda, se o profissional que prestou a assistência não foi o mesmo que preencheu a declaração de óbito ${ }^{11}$. 0 médico não assistente possui obviamente menos informações e conhecimento sobre o paciente, desfavorecendo o preenchimento completo.

Quanto ao conhecimento do documento do Ministério da Saúde e do Conselho Federal de Medicina, verificou-se que $47,02 \%$ o conheciam. Este resultado aproxima-se do visto por outros autores ${ }^{12}$ que entrevistaram 18 médicos e apenas $40 \%$ afirmaram conhecer o documento. Dessa forma, apesar de haver essa bem elaborada instrução, o desconhecimento de sua existência é bastante alto, dificultando o preenchimento adequado.

Em relação ao uso de termos não recomendados - como "parada cardíaca", "parada cardiorrespiratória" ou "falência múltipla de órgãos" - para o preenchimento de causa básica nas declarações de óbito, parte dos médicos $(38,2 \%)$ declarou fazer uso. No presente estudo foram entrevistas apenas $34(6,43 \%)$ DO com uso de termos vagos ou inadequados, sendo a "parada cardiorrespiratória" em 15 $(2,84 \%)$ documentos a ocorrência mais frequente.

O documento do CFM/MS ${ }^{2}$ desestimulou o uso desses termos no preenchimento das causas básicas do óbito. Outras pesquisas apresentaram índices mais elevados de termos vagos ou inadequados. O citado estudo realizado em Belo Horizonte demonstrou que os termos "choque" (35\%), "insuficiência respiratória" (30\%), "parada cardiorrespiratória" (10\%) e "falência múltipla de órgãos" (5\%) foram utilizados como causas básicas de morte ${ }^{12}$. Na pesquisa realizada em Belém a utilização de termos vagos - "insuficiência respiratória", "parada cardiorrespiratória" e "falência múltipla de órgãos" - foi localizada em $55 \%$ das DO ${ }^{9}$, bastante superior ao verificado na presente pesquisa. Na África do Sul, um estudo realizado em 304 de- clarações de óbitos hospitalares deparou com o diagnóstico inadequado de "parada cardiorrespiratória" em número ainda maior: $78,9 \%$ dos $\operatorname{casos}^{13}$.

$\mathrm{Na}$ análise dos dados, ficou evidenciado que a maioria dos médicos participantes $(58,53 \%)$ relatou dificuldades no preenchimento da declaração em caso de óbito sem assistência médica e nos itens relativos à causa básica, intermediária e imediata ou terminal da morte. Resultado similar foi visto no estudo desenvolvido em Belo Horizonte ${ }^{12}$, no qual $68 \%$ dos médicos relataram alguma dificuldade no preenchimento. Naquele trabalho as principais dificuldades apontadas foram: grande quantidade de campos a serem preenchidos (31\%), morte violenta (16\%), explicações pouco claras sobre o preenchimento (11\%), imposição de cartórios (5\%) e preenchimento na condição de médico substituto (5\%).

Em relação ao conhecimento sobre DO adquirido durante a graduação, aproximadamente a metade dos médicos considerou bom ou muito bom (47\%). Os demais o classificaram como aceitável, ruim ou muito ruim, evidenciando a necessidade de enfatizar o conhecimento desse estudo durante a graduação médica. Não houve diferenças de conhecimento sobre o preenchimento das declarações de óbito quando se comparou os grupos por tempo de formação $(p=0,250)$. Entretanto, deve ser levado em consideração o viés advindo do componente subjetivo de uma autoavaliação, haja vista que outros estudos apontaram para problemas mais frequentes no preenchimento feito por médicos mais jovens ${ }^{14,15}$.

Estudo desenvolvido em Fortaleza ${ }^{16}$ evidenciou expressiva redução das causas mal definidas nas declarações de óbitos de 2003 a 2008. Na premissa de aperfeiçoar o preenchimento das DO, e consequentemente as informações do SIM, recomendou maior integração entre serviço e ensino médico, propiciando melhor aprendizado do aluno e entendimento da importância dos dados epidemiológicos. Além disso, o estudo propõe integrar os diferentes sistemas de informação disponíveis, compor um grupo técnico para análise do SIM e promover parcerias com o conselho regional de medicina e secretarias de saúde.

No âmbito internacional, estudo realizado com 317 médicos do Qatar ${ }^{14}$ verificou que apenas $22,7 \%$ haviam recebido treinamento anterior sobre declaração de óbito e as principais dificuldades que apontaram foram o preenchimento em casos complicados $(47,3 \%)$ e a falta de treinamento $(43,55 \%)$. Outro interessante estudo realizado na Austrália ${ }^{17}$ sugere a realização de workshops para treinamento dos médicos no preenchimento de declarações de óbito. 
A pesquisa mostrou que o índice de acertos passou de $33,3 \%$, antes do treinamento, para $69,2 \%$ após o treinamento. Em Taiwan ${ }^{15}$, uma pesquisa inferiu sugestão semelhante, mas os autores evidenciaram a necessidade de promover treinamento direcionado aos médicos mais jovens e de hospitais com baixa ou nenhuma atividade de ensino, onde a deficiência de preenchimento era maior. Neste amplo estudo foram analisadas 4.123 declarações de óbito, das quais somente $61 \%$ estavam corretamente preenchidas.

Estudo realizado em Recife ${ }^{18}$ aventou que a deficiência de qualidade do preenchimento dos registros contidos nas DO remonta ao curso de graduação médica, decorrendo da pouca importância creditada ao conhecimento dos dados como fonte de informação para a saúde da população. Essa distorção prejudica a compreensão dos registros gerados por esse valioso instrumento de coleta de informações.

Dessa forma, presume-se como necessário, durante a graduação, a pós-graduação e o exercício profissional, propiciar maior quantidade de informação aos médicos sobre a importância da declaração de óbito, ensinando-lhes, sobretudo, como preenchê-la correta e completamente, seja no detaIhamento da causa básica de morte, seja na sequência de eventos e no uso de termos precisos.

Embora o preenchimento do médico seja designado na Lei 6.015/73 como atestado, a designação do documento contendo as informações completas a serem encaminhadas ao cartório e à secretaria municipal de saúde foi consagrada com a denominação declaração de óbito pela Lei 11.976/09 4,19. A necessidade de maior ênfase ao ensino sobre a declaração de óbito, para seu correto preenchimento, ficou evidente nesta lei ${ }^{4}$ que enfatiza tanto a origem médica do documento como sua finalidade estatística, cujo interesse médico-sanitário é destacado por vários autores ${ }^{19,20}$. Para maior amplitude de efeito e eficácia no preenchimento, a Resolução CFM 1.779/05 ${ }^{21}$ especificou que a declaração de óbito seja preenchida pelo médico assistente ou substituto, também podendo ser fornecida por um médico designado pela instituição e, em caso de morte violenta ou suspeita, pelos médicos legistas.

As falhas identificadas no preenchimento apontaram para a necessidade de maior incentivo do estudo das declarações de óbito durante a graduação ${ }^{14,15}$ e pós-graduação, bem como de se promover atividade relacionada em eventos para médicos, cujos resultados são efetivos mesmo quando cursados uma única vez ${ }^{16,17}$. A alegação feita por alguns médicos de que o documento é de pre- enchimento complicado pode ser atribuída à pouca informação recebida. No entanto, o preenchimento, cada vez em maior número, por médicos não assistentes pode estar constituindo uma dificuldade superveniente que contribui para o preenchimento incompleto ou inadequado das DO.

O esforço conjunto do CFM e do MS para elaborar documento voltado a orientar e normatizar ${ }^{2}$, em linguagem simples, visou fortalecer o compromisso do médico com o correto e importante preenchimento da declaração de óbito. Contudo, para que este objetivo seja alcançado, a divulgação dessa orientação precisa ocorrer com maior intensidade, não somente na graduação e pós-graduação, mas também a todos os médicos no exercício da profissão.

\section{Considerações finais}

A metade das declarações de óbito analisadas estava incompletamente preenchida e, ao contrário do esperado, houve piora do número de preenchimentos completos durante o ano 2011 em relação ao ano 2009 ( $p<0,001)$.

Os resultados desta pesquisa contrastam com a importância epidemiológica do preenchimento desses dados para as políticas públicas de saúde do país e apontam para a necessidade de se aprimorar o ensino sobre a declaração de óbito. Infere-se que a abordagem deste tema durante a graduação médica deva ocorrer não somente em Medicina Legal, mas longitudinalmente no teor de outras disciplinas, incluindo-se a Ética Médica pelos aspectos morais envolvidos no preenchimento. Neste contexto, a orientação formulada pelo CFM/MS - denominada A declaração de óbito: documento necessário e importante ${ }^{2}$ - insere-se como uma fonte de estudos para a graduação e de consulta para os profissionais. Propõe-se ainda que os conselhos de medicina, Ministério da Saúde e outras instituições afins promovam em seus eventos workshops sobre o preenchimento de declaração de óbito - cuja eficácia no índice de acertos transparece nos resultados dos testes de quem participa uma única vez.

Em conclusão, o ensino sobre a declaração de óbito durante a graduação médica, pós-graduação e exercício profissional do médico precisa ser incentivado e as orientações existentes serem divulgadas com maior eficácia. Outros estudos serão necessários para identificar as falhas na formação médica e as dificuldades que ocorrem durante o exercício profissional, para que sejam apontadas soluções mais abrangentes. 


\section{Referências}

1. Brasil. Ministério da Saúde. Fundação Nacional de Saúde. Manual de procedimentos do Sistema de Informações sobre Mortalidade. Brasília: Funasa; 2001.

2 Brasil. Ministério da Saúde. Conselho Federal de Medicina. Centro Brasileiro de Classificação de Doenças. A declaração de óbito: documento necessário e importante. $3^{\mathrm{a}}$ ed. Brasília: MS; 2009.

3. Laurenti R, Jorge MHPM. O atestado de óbito. São Paulo: Centro Brasileiro de Classificação de Doenças; 1985. (Série Divulgação, $n^{\circ} 1$ )

4. Brasil. Lei $n^{\circ} 11.976$, de 8 de julho de 2009. Dispõe sobre a declaração de óbito e a realização de estatísticas de óbitos em hospitais públicos e privados. Diário Oficial da União. 8 jul. 2009;Seçao $\mathrm{I}: 1$.

5. Conselho Federal de Medicina. Resolução n 1.995, de 17 de setembro de 2009. Aprova o Código de Ética Médica. Diário Oficial da União. 24 set. 2009; Seção I:90.

6. Tólio RC, Swiech TI, Moritz RD, Bonamigo EL. Avaliação sobre a sedoanalgesia durante os momentos finais da vida. [TCC]. [Internet]. Joaçaba: Universidade do Oeste de Santa Catarina; 2010 (acesso 5 mar. 2014). Disponível: http://unoesc.edu.br/mala/avaliacaosedoanalgesia.pdf

7. Niobey FML, Cascão AM, Duchiade MP, Sabroza PC. Qualidade do preenchimento de atestados de óbitos em menores de um ano na região metropolitana do Rio de Janeiro. Rev Saúde Pública. 1990;24(4):311-8.

8. Stuque CO, Cordeiro JA, Cury PM. Avaliação dos erros ou falhas de preenchimento dos atestados de óbito feitos pelos clínicos e pelos patologistas. J Bras Patol Med Lab. 2003;39(4):361-4.

9. Silva JA, Yamaki VN, Oliveira JP, Teixeira RK, Santos FA, Hosoume VS. Declaração de óbito, compromisso no preenchimento: avaliação em Belém, Pará, Brasil, em 2010. Rev Assoc Med Bras. 2013;59(4):335-40.

10. Paes NA. Qualidade das estatísticas de óbitos por causas desconhecidas dos estados brasileiros. Rev Saúde Pública. 2007;41(3):436-45.

11. Sousa MH, Cecatti JG, Hardy EE, Serruya SJ. Morte materna declarada e o relacionamento de sistemas de informação em saúde. Rev Saúde Pública. 2007;41(2):181-9.

12. Mendonça FM, Drummond E, Cardoso AMP. Problemas no preenchimento da declaração de óbito: estudo exploratório. Rev Bras Estud Popul. 2010;27(2):285-95.

13. Meel BL. Certification of deaths at Umtata General Hospital, South Africa. J Clin Forensic Med. 2003;10(1):13-5.

14. Al-Kubaisi NJ, Said $H$, Horeesh NA. Death certification practice in Qatar. Public Health. 2013;127(9):854-9.

15. Lu TH, Shau WY, Shih TP, Lee MC, Chou MC, Lin CK. Factors associated with errors in death certificate completion: a national study in Taiwan. J Clin Epidemiol. 2001;54(3):232-8.

16. Pinto CHCC, Gurgel MGI, Alves MDS, Passos MLL, Diniz SAN, Conceição MR. Investigação de óbitos por causas mal definidas: estratégia de fortalecimento da vigilância em saúde. Rev Rene. 2012;13(4):871.

17. Rampatige R, Wainiqolo I, Singh S, Riley I. Engaging physicians in improved cause of death certification: evaluation of an education intervention. Lancet. 2013;381:S122.

18. Vanderlei LC, Arruda BKG, Frias PG, Arruda S. Avaliação da qualidade de preenchimento das declarações de óbito em unidade terciária de atenção à saúde materno-infantil. Inf Epidemiol SUS. 2002;11(1):7-14.

19. Lopes JCN. Aspectos éticos e jurídicos da declaração de óbito. Rev. bioét. (Impr.). 2011;19(2):36782.

20. França GV. Direito médico. $9^{\mathrm{a}}$ ed. rev. atual. Rio de Janeiro: Forense; 2007.

21. Conselho Federal de Medicina. Resolução CFM n 1.779/2005. Regulamenta a responsabilidade médica no fornecimento da declaração de óbito. Revoga a Resolução CFM n 1.779, de 11 de novembro de 2005. Regulamenta a responsabilidade médica no fornecimento da declaração de óbito. Revoga a Resolução CFM n 1.601/2000. Diário Oficial da União. 5 dez. 2005; Seção I:121.

\section{Participação dos autores}

Luan Lucena e Gustavo Henrique Bocalon Cagliari participaram do desenho do estudo, da coleta e interpretação dos dados e redação do artigo. Julio Tanaka coorientou a pesquisa, participou da interpretação dos dados e redação do artigo. Elcio Luiz Bonamigo coordenou a pesquisa, participou do desenho do estudo, da interpretação dos dados e redação do artigo.

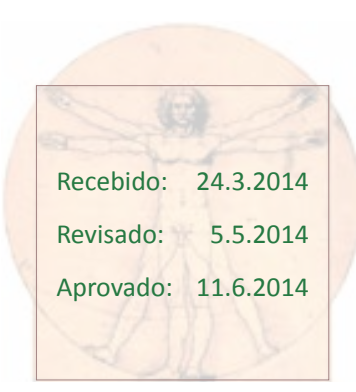

